\title{
Success Rate of Airway Management by Residents in a Pre-hospital Emergency Setting: a Retrospective Study
}

\author{
Eric Albrecht ${ }^{1,2}$, Bertrand Yersin', Donat R. Spahn², Daniel Fishman', Olivier Hugli ${ }^{1}$
}

\begin{abstract}
Objective: The objective of this retrospective study over a 5-year period was to assess the success rate of airway management by residents. Criteria of successful airway management were both the adherence to a standardized protocol of pre-hospital airway management and successful endotracheal intubation (ETI) in rescue missions.

Methods: The minimal level of training time required for residents rotating in the pre-hospital emergency team was either 1 year in our university department of anesthesiology, or 3 years of internal medicine including 20 ETIs under supervision in the operating room. According to a strict protocol detailing indications and drugs to be administered, residents performed rapidsequence intubation (RSI) except in cases of cardiopulmonary arrests where ETI was performed without drugs. Adherence to the protocol of airway management was evaluated according to data provided by the residents. Successful endotracheal tube placement was confirmed only in transported patients with a combination of clinical signs, infrared capnography, and a chest X-ray on hospital admission.

Results: A total of 13,537 rescue missions were reviewed. The protocol adherence was 96.1\%. ETI was attempted in 753 patients, and successful placement was confirmed in $98.2 \%$.

Conclusion: Pre-hospital airway management (protocol adherence and proper endotracheal tube
\end{abstract}

placement) was successful overall in $94.3 \%$ of rescue missions. Our results support the efficacy of a pre-hospital emergency rescue system reinforced by residents.

\section{Key Words}

Airway management - Orotracheal intubation · Medical emergency team

Eur J Trauma 2006;32:516-22

DOI 10.1007/s00068-006-6001-z

\section{Introduction}

The goal of early airway control is to reduce morbidity and mortality among patients sustaining severe trauma or significant medical emergencies [1]. It is indeed the A step of advanced life support, and endotracheal intubation (ETI) is furthermore considered the "gold standard" of emergency airway control in terms of quality and safety. Since the first formal report in 1988 [2], rapid sequence induction (RSI) has been used with increasing frequency in pre-hospital emergency settings in order to facilitate ETI and airway control, but its use remains controversial. The published range of ETI success in the pre-hospital setting varies between 49 and 99\% [3-9], and is partially dependent on the performer's level of training. This wide range of success contributes to the controversy.
'Emergency Department, Centre Hospitalier
Universitaire Vaudois (CHUV), Lausanne, Switzerland,
${ }^{2}$ Department of Anaesthesiology, Centre Hospitalier
Universitaire Vaudois (CHUV), Lausanne, Switzerland.

Received: January 2, 2006; revision accepted: February 3, 2006 
In the Anglo-American model, emergency medical technicians or paramedics usually provide pre-hospital care and airway management during ground transport rescue, while nurses, emergency physicians or residents are involved in air rescue missions [4, 6-12]. In the Franco-German model, pre-hospital care and airway management are performed by emergency physicians [5].

Several curricula for emergency medicine residents have been designed to offer a field experience. In the United States of America, the Residency Review Committee also requires residency programs to offer such a structured experience. An in-field pre-hospital experience appeared valuable to residents because it offered an opportunity to perform field procedures [13], providing exposure to critically ill or injured patients, and improving field research [14]. However, no recent studies have investigated the residents' success rate in performing such a crucial procedure as airway management.

In Switzerland, emergency medicine is not a specialty. Emergency departments are staffed by residents from internal medicine, surgery, pediatrics and anesthesiology working under the supervision of attending physicians. Pre-hospital medical care is provided primarily by internal medicine and anesthesiology residents who perform 3-12-month rotations. Before the beginning of such a rotation, each resident benefits from a specific training in emergency medicine protocols and procedures. They are instructed strict protocols of airway control including ETI and RSI.

The objectives of this retrospective study were to assess the adherence of residents to a standardized protocol of pre-hospital airway management, and to assess their success rate of ETI.

\section{Materials and Methods}

This study was reviewed and approved by ethics committee at our institution.

Our University Hospital is a Level 1 trauma center with 950 acute beds. The annual census of the Emergency Department shows approximately 40,000 admissions. The pre-hospital emergency medical service serves a population of 800,000 inhabitants. Depending on alarm keywords and locations of the emergency, patients are rescued either by ambulance or by helicopter. The ambulance team is composed of one rescue technician and one resident. The helicopter team is composed of a pilot, a rescue technician, and a resident. The training
Table 1. Protocol of indications of pre-hospital intubation.

1) Head trauma with Glasgow coma scale $\leq 8$

2) Severe facial trauma

3) Multiply injured patient with shock (systolic blood pressure $<90 \mathrm{mmHg}$ )

4) Burned patient with inhalation syndrome

5) Respiratory failure with confusion (respiratory rate $>35 / \mathrm{min}$ and saturation $<90 \%$ )

6) Cardiac arrest

for the rescue technician is a 3-year course, which provides lectures, manikin sessions, and practical training in the field. Rescue technicians insert peripheral venous catheters, inject drugs, but are not certified in advanced life support. Only residents are allowed to perform endotracheal intubation.

The minimal level of training required for residents rotating in the pre-hospital emergency team consists of either 1 year of residency in our anesthesiology department, or 3 years of internal medicine. Before residents are allowed to work in our pre-hospital emergency service, they are trained and required to demonstrate expertise with pre-hospital advanced cardiac and traumatic life support, including ETI on manikins during a 3-day course. Internal medicine residents must have performed in addition a minimum of 20 ETI under supervision in the operating room. The pre-hospital emergency rotation lasts 3-12 months. The resident staff is composed of four anesthesiology residents staffing both helicopters and ambulances, and two internal medicine residents staffing only ambulances. They are on call for $24 \mathrm{~h}$ every other day.

Residents follow strict indications for pre-hospital ETI based on the protocol described in Table 1. ETI is performed with RSI, except in cases of cardiopulmonary arrest. The protocol of RSI is described in Table 2 [15]. ETI is always performed via the oral route. If ETI fails, laryngeal mask placement is attempted; if the latter fails, the patient is ventilated by facial mask during transportation. A transtracheal catheter [16] and a cricothyrotomy set are available if facial mask ventilation is impossible. On site, successful ETI is confirmed by the absence of air sounds in the stomach on auscultation, symmetric thorax ventilation with bilateral breath sounds, and endtidal $\mathrm{CO}_{2}$ measured by infrared capnography, which is continued during transportation. Once the patient is intubated and transported to the hospital, the hospital resuscitation team leader verifies the successful 
Table 2. Protocol of pre-hospital rapid sequence intubation.

1) Equipment and suction device check

2) Manual in line stabilization if cervical spine injury is suspected

3) Cricoid pressure (Sellick)

4) Premedication with lidocaine (1 $\mathrm{mg} / \mathrm{kg}$ iv) if head trauma with

Glasgow coma scale $\leq 8$

5) Induction with etomidate $(0.3 \mathrm{mg} / \mathrm{kg}$ iv)

6) Neuromuscular blocker with succinylcholine $(1.5 \mathrm{mg} / \mathrm{kg}$ iv)

7) Hyperventilation for 60 seconds

8) Intubation with manual in line stabilization and cricoid pressure: maximum of 3 attempts

Endotracheal tube placement confirmation by auscultation; cricoid pressure release; tube secured; if unsuccessful, laryngeal-

9) mask then facial mask ventilation; if ventilation impossible, perform transtracheal ventilation via transtracheal catheter or cricothyrotomy route

10) Semirigid cervical collar if cervical spine injury is suspected

11) Sedation with midazolam (0.1 mg/ $\mathrm{kg}$ iv)

12) Analgesia with fentanyl (up to $5 \mu \mathrm{g} / \mathrm{kg}$ iv)

13) Prolonged neuromuscular block with vecuronium $(0.1 \mathrm{mg} / \mathrm{kg})$ placement of the endotracheal tube by clinical evaluation, infrared capnography, and chest X-ray.

After each mission, residents fill in a standardized data sheet containing the patient's demographic data, vital signs before and after ETI, suspected diagnosis, success of peripheral venous access, drugs administered, ETI indication, ETI position on arrival at hospital, Injury Severity Score, hospital diagnosis, and finally the outcome at $48 \mathrm{~h}$. An attending physician conducts a critical assessment of each mission with the resident through a systematic review of all data sheets. A complete filling of all details in the data sheets is verified by the attending physician. Difficult interventions are discussed on weekly rounds. One day a week, the attending physician supervises residents in the field. A data manager files all the information in a database once the forms have been reviewed and checked for completion by the attending physician.

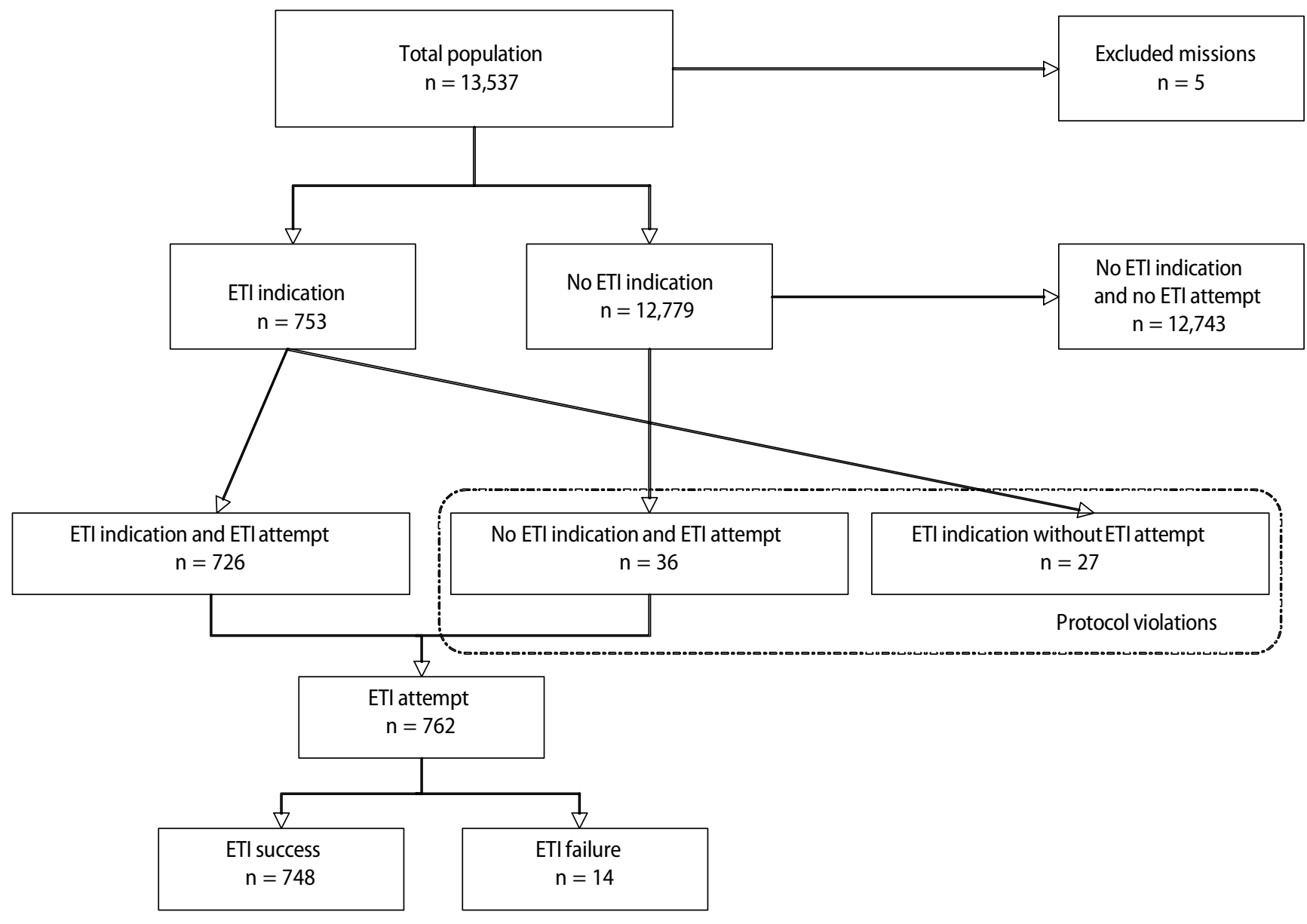

Figure 1. The study profile. 
Table 3. Patient characteristics.

\begin{tabular}{|c|c|c|c|c|c|}
\hline & $\begin{array}{l}\text { Study } \\
\text { popula- } \\
\text { tion }\end{array}$ & $\begin{array}{l}\text { ETI at- } \\
\text { tempt }\end{array}$ & $\begin{array}{l}\text { ETI at- } \\
\text { tempt and } \\
\text { ETI } \\
\text { indication }\end{array}$ & $\begin{array}{l}\text { ETI at- } \\
\text { tempt } \\
\text { without } \\
\text { indication }\end{array}$ & $\begin{array}{l}\text { No ETI } \\
\text { attempt } \\
\text { despite } \\
\text { indication }\end{array}$ \\
\hline Number & 789 & 762 & 726 & 36 & 27 \\
\hline $\begin{array}{l}\text { Age }(\text { years })(\text { mean } \pm S D) \\
(\text { minimum-maximum })\end{array}$ & $\begin{array}{l}51 \pm 24 \\
(1-102)\end{array}$ & $\begin{array}{l}51 \pm 23 \\
(1-94)\end{array}$ & $\begin{array}{l}51 \pm 23 \\
(1-94)\end{array}$ & $\begin{array}{l}57 \pm 21 \\
(18-93)\end{array}$ & $\begin{array}{l}42 \pm 31 \\
(1-102)\end{array}$ \\
\hline Sex (male/female) & $556 / 233$ & $535 / 227$ & $513 / 213$ & $22 / 14$ & $21 / 6$ \\
\hline \multicolumn{6}{|l|}{ Indication for intubation } \\
\hline $\begin{array}{l}\text { Head trauma with Glasgow } \\
\text { coma scale } \leq 8\end{array}$ & 289 & 277 & 277 & - & 12 \\
\hline Severe facial trauma & 29 & 29 & 29 & - & - \\
\hline $\begin{array}{l}\text { Multiply injured patient } \\
\text { with shock (systolic blood } \\
\text { pressure }<90 \mathrm{mmHg} \text { ) } \\
\text { (systolic blood pressure } \\
<90 \mathrm{mmHg} \text { ) }\end{array}$ & 19 & 18 & 18 & - & 1 \\
\hline $\begin{array}{l}\text { Burn patient with inhalation } \\
\text { syndrome }\end{array}$ & 13 & 12 & 12 & - & 1 \\
\hline $\begin{array}{l}\text { Respiratory failure with con- } \\
\text { fusion (respiratory rate } 35 / \\
\text { min and saturation }<90 \% \text { ) }\end{array}$ & 145 & 136 & 136 & - & 9 \\
\hline Cardiac arrest & 258 & 254 & 254 & - & 4 \\
\hline \multicolumn{6}{|l|}{ Injury Severity Score } \\
\hline $1-15$ & 69 & 67 & 61 & 4 & 4 \\
\hline $16-30$ & 166 & 162 & 154 & 3 & 9 \\
\hline$>30$ & 123 & 127 & 121 & 1 & 1 \\
\hline
\end{tabular}

In this study, all patients with ETI attempt or ETI indications were included in the study. Airway management was considered successful if there was no protocol violation and no ETI placement failure. Pre-hospital protocol violation was present in two conditions: ETI not attempted despite ETI indication, and ETI attempted without ETI indication. We also reviewed all cases of patients intubated within $15 \mathrm{~min}$ of arrival to the emergency department as possible cases of non-respect of pre-hospital ETI indication. We although reviewed all those ETI-attempted cases without initial indication to verify that the ETI was not justified by deterioration of the patient's condition and performed during transportation.

ETI placement failure was defined as any of the following conditions: (1) the inability to intubate the airway requiring ventilation with a laryngeal mask, facial mask, or a transtracheal catheter, (2) esophageal placement of the endotracheal tube recognized only in the emergency room (3) failed intravenous access for patients who required RSI. Only patients who were transported, and in whom the position of the endotracheal tube could be verified upon hospital admission, were included. All cases of failed ETI were reviewed for specific causes.
Data were stored using Microsoft Access 5.0 (Microsoft, Inc., Redmond, Washington, USA). Statistical analysis was completed using Sigma stat 2.03 (Access Softake Inc., Chicago, IL, USA), and Microsoft Excel 2000 (Microsoft, Inc.). The results are presented in a descriptive fashion with mean \pm SD [minimum-maximum]. Chi-square test was used to compare ETI success between trauma patients and medical patients.

\section{Results}

From January 1, 1996 until November 30, 2001, 13,537resident-staffed ambulance or helicopter missions were analyzed. Only five missions were excluded: two lacked documentation of ETI status, and three patients were found in a state of rigor mortis. The study profile is depicted in Figure 1. Seventy-four residents rotated through our pre-hospital emergency team during the study period. On average, each resident performed $11 \pm 4$ intubations (3-16 [minimum-maximum]).

\section{Adherence to the Protocol}

Among the remaining 13,532 missions studied, 789 cases of ETI indications $(n=753)$ or ETI attempts without ETI indications $(n=36)$ were assessed. The patients' demographic data are presented in the first column of Table 3. Patients were middle-aged, mostly male. Pediatric cases represented $6.1 \%$ of all missions (48/789). Indications for ETI were cardiac arrest in 34.0\% (258/753), respiratory failure in $19.0 \%(145 / 753)$ and trauma in $47.0 \%$ (350/753). The etiology of cardiac arrest was non-traumatic in $88.8 \%$ of patients. Among 350 traumas, 240 were blunt $(68.6 .0 \%), 9$ penetrating $(2.6 \%)$, 88 isolated head trauma (25.1\%), and 13 burns (3.7\%).

Among patients with ETI indication, ETI was not attempted in 27 patients or 3.6\% (27/753). Ten had ETI in the emergency department within 15 min of arrival. Among the 17 remaining patients, 6 were over 80 years old and suffering from several co-morbidities or were 
Table 4. Characteristics of patients with failed intubation.

\begin{tabular}{|c|c|c|c|c|c|}
\hline Patient & $\begin{array}{l}\text { Age } \\
\text { (years) }\end{array}$ & $\begin{array}{l}\text { Indication of } \\
\text { intubation }\end{array}$ & $\begin{array}{l}\text { Type of intu- } \\
\text { bation failure }\end{array}$ & $\begin{array}{l}\text { Rescue strategy } \\
\text { ventilation }\end{array}$ & $\begin{array}{l}\text { Hospital diagnosis / } \\
\text { Injury Severity Score }\end{array}$ \\
\hline 1 & 43 & Cardiac arrest & ETI failure & Facial mask & Acute coronary syndrome \\
\hline 2 & 76 & Cardiac arrest & ETI failure & Laryngeal-mask & Acute coronary syndrome \\
\hline 3 & 67 & None & ETI failure & Facial mask & Cerebral hemorrhage \\
\hline 4 & 89 & None & ETI failure & Facial mask & Epilepsy \\
\hline 5 & 49 & None & ETI failure & Facial mask & Epilepsy \\
\hline 6 & 56 & None & ETI failure & Laryngeal-mask & Epilepsy \\
\hline 7 & 57 & Cardiac arrest & $\begin{array}{l}\text { Esophageal } \\
\text { intubation }\end{array}$ & - & Acute coronary syndrome \\
\hline 8 & 32 & None & ETI failure & Facial mask & Isolated head injury / 17 \\
\hline 9 & 69 & Head trauma & ETI failure & Laryngeal-mask & Penetrating trauma / 75 \\
\hline 10 & 35 & Burned patient & ETI failure & Laryngeal-mask & Burn / 25 \\
\hline 11 & 66 & Head trauma & ETI failure & Laryngeal-mask & Isolated head injury / 25 \\
\hline 12 & 69 & Head trauma & ETI failure & Facial mask & Isolated head injury / 25 \\
\hline 13 & 22 & Head trauma & $\begin{array}{l}\text { Intravenous } \\
\text { access failure }\end{array}$ & Facial mask & Blunt trauma / 22 \\
\hline 14 & 27 & Cardiac arrest & $\begin{array}{l}\text { Intravenous } \\
\text { access failure }\end{array}$ & Facial mask & Blunt trauma / 36 \\
\hline
\end{tabular}

age. The last 2 of 14 patients had intravenous access failure preventing RSI and were ventilated with a facial mask. Neither transtracheal ventilation nor cricothyrotomy were performed. Interestingly, 5 patients among these 14 cases had no ETI indication: 3 cases of epilepsy, 1 cerebral hemorrhage, and 1 head trauma with Glasgow coma scale score $>8$. These five patients were counted twice in the calculation of the overall success of airway management as they have both protocol violation and ETI placement failure. Fourteen different residents were involved in these failed ETI. Finally ETI success rate was $97.9 \%$ (329/336) among trauma patients and $98.4 \%$ (419/426) among medi-

in terminal phase of cancer. The last 11 patients could have been treated without requiring ETI.

Among 12,779 patients without ETI indication, $36(0.3 \%)$ had nevertheless an ETI attempt. This group included 2 hypothermia, 15 cerebral hemorrhages, 8 traumas without shock, 2 drug intoxications, 4 epilepsy, 1 COPD, 1 pneumonia, 2 acute coronary syndromes, and 1 cardiac arrhythmia. Humane reasons was the justification given by the residents for violating the protocol in two cases of a traumatic amputation.

In summary, the overall $3.9 \%$ cumulative protocolviolation rate was the sum of the $3.6 \%$ group of no ETI attempt despite ETI indication and $0.3 \%$ group of ETI attempt without ETI indication.

\section{ETI Placement Success Rate}

Among the 762 eligible patients (726 patients with ETI indications and ETI attempts +36 patients without ETI indications but with ETI attempts), 748 (98.2\%) were successfully intubated. This included 17 (2.3\%) right mainstem bronchus intubations recognized in the emergency room. ETI was unsuccessful in 14 patients (Table 4). In 11 of 14 cases, attempts to intubate the trachea failed, and consequently 5 patients required laryngeal-mask ventilation; the 6 remaining patients had facial mask ventilation. One of 14 patients with cardiac arrest suffered from an esophageal intubation recognized only in hospital. He survived, and suffered no neurological dam- cal patients $(\mathrm{p}=0.79)$. There was no ETI placement failure among pediatric patients.

\section{Discussion}

Endotracheal intubation in the pre-hospital setting is a challenging procedure due to the uncontrolled environment. This single-site retrospective study of a large cohort of patients describes the performance of a prehospital resident-reinforced rescue system. Both adherence to the protocols and ETI success rate were assessed and found to be high.

ETI is associated with numerous complications, and must be used only in situations where benefits outweigh the risks. Guidelines present the evidence supporting the beneficial use of ETI. Protocols are one way to improve physicians' compliance with these guidelines, ensuring the delivery of the best possible level of care. Unfortunately, studies on ETI success rate report rarely protocol-violation rates. In a pediatric population, Gausche et al. [6] reported a protocol-violation rate of $3 \%$, similar to ours. Our protocol-violation rate was low, and some of the protocol violations were based on sound medical or humane reasons. Therefore, true cases of underuse or overuse of ETI were even less frequent. Continual critical assessment such as a systematic review of reports and weekly rounds are an essential part of the medical teaching process, and may be one of the reasons for this low protocol-violation rate. 
Our ETI placement success rate was $98.2 \%$. Proper tube placement was not only confirmed by the resident but also by the resuscitation team leader in the emergency department using multiple independent techniques. In our case series, there was only one esophageal intubation. Interestingly, this patient survived, which might suggest that the endotracheal tube was dislodged during transportation. Improper securing of the tube rather than ETI placement failure thus may have been the problem in this case. Adequate training, and the constant use of multiple-tube confirmation techniques already in the pre-hospital setting contribute to such a low incidence. However, similar high success rates have been described for ETI with RSI in a pre-hospital setting $[5,11,12]$. For example, in the series of Adnet et al. [5], RSI success rate was $99 \%$. But unlike in our study, only senior physicians performed the RSI.

In a recent study, inadequate muscle relaxation was found as one of the major predictors of ETI placement failure, providing support for the use of an RSI protocol [17]. The advantage of RSI is to enable pre-hospital providers to achieve successful intubation rates similar to those seen in the in-hospital setting despite an incidence of difficult intubation three to five times higher [18-20]. This advantage of RSI may have contributed to the success rate of our study.

The overall success rate of airway management in our study was $94.3 \%$. Failure of airway management is explained either by protocol violation $(3.9 \%)$ or intubation failure $(1.8 \%)$. The failed intubations were performed by eight anesthesiologist residents and six internal medicine residents. As the exact number of missions and intubations performed by residents of each specialty is not available in our database, we were unable to assess if one type of residency program was associated with a different pre-hospital ETI success rate. Nevertheless, 1 year of anesthesiology residency or 3 years of internal medicine with 20 ETI performed under supervision in the operating room seemed to be sufficient in order to efficiently acquire the skills required in the pre-hospital field. Moreover, these skills were maintained since every resident had the opportunity to perform about 11 intubations in the field. This high number contrasts with other studies where paramedics perform an RSI every $1-4$ years $[21,22]$. An additional benefit of our resident-based system is to provide a valuable experience in pre-hospital care and the diversity of situations that it encompasses.

Interestingly, ETI success rate was not lower in the trauma than in the medical group. This is unexpected, as specific lesions of trauma patients could make ETI more difficult: severe facial trauma, cervical spine injury with manual in-line stabilization, burned patients with inhalation syndrome.

This study has several potential limitations. First, information bias may cause a differential misclassification of patients. As data sheets were filled by the residents after each mission, their decision to attempt ETI or not could be justified a posteriori. But review of the objective clinical information provided by rescue technicians in the field minimized this bias. Secondly, we have no record of the number of intubation attempts made per patient, of the oxygen saturation during the attempts, or of complications of intubation other than the tube malpositioning. These adverse events would be expected to increase morbidity or mortality, but not our outcomes of interest, i.e., protocol adherence and ETI success rates.

In conclusion, this data of a resident-reinforced rescue system needs to be confirmed by a prospective study addressing the limitations of the current retrospective analysis before its implementation can be recommended. Eventually, a resident-based out-of-hospital rescue system may be considered as an effective and educational alternative to systems based on paramedics or professional emergency physicians.

\section{References}

1. Pepe PE, Copass MK, Joyce TH. Prehospital endotracheal intubation: rationale for training emergency medical personnel. Ann Emerg Med 1985;14:1085-92.

2. Hedges JR, Dronen SC, Feero S, et al. Succinylcholine-assisted intubations in prehospital care. Ann Emerg Med 1988;17:469-72.

3. Bradley JS, Billows GL, Olinger ML, et al. Prehospital oral endotracheal intubation by rural basic emergency medical technicians. Ann Emerg Med 1998;32:26-32.

4. Jacobs LM, Berrizbeitia LD, Bennett B, et al. Endotracheal intubation in the prehospital phase of emergency medical care. JAMA 1983;250:2175-7.

5. Adnet F, Jouriles NJ, Le Toumelin P, et al. Survey of out-of-hospital emergency intubations in the French prehospital medical system: a multicenter study. Ann Emerg Med 1998;32:454-60.

6. Gausche M, Lewis RJ, Stratton SJ, et al. Effect of out-of-hospital pediatric endotracheal intubation on survival and neurological outcome: a controlled clinical trial. JAMA 2000;283:783-90.

7. Winchell RJ, Hoyt DB. Endotracheal intubation in the field improves survival in patients with severe head injury. Trauma Research and Education Foundation of San Diego. Arch Surg 1997;132:592-7.

8. Eckstein M, Chan L, Schneir A, et al. Effect of prehospital advanced life support on outcomes of major trauma patients. J Trauma 2000;48:643-8.

9. Katz SH, Falk JL. Misplaced endotracheal tubes by paramedics in an urban emergency medical services system. Ann Emerg Med 2001;37:32-7. 
10. Sayre MR, Sakles JC, Mistler AF, et al. Field trial of endotracheal intubation by basic EMTs. Ann Emerg Med 1998;31:228-33.

11. Sloane C, Vilke GM, Chan TC, et al. Rapid sequence intubation in the field versus hospital in trauma patients.J Emerg Med 2000;19:259-64.

12. Slater EA, Weiss SJ, Ernst AA, et al. Preflight versus en route success and complications of rapid sequence intubation in an air medical service. J Trauma 1998;45:588-92.

13. Houry DE, Pons PT. The value of the out-of-hospital experience for emergency medicine residents. Ann Emerg Med 2000; 36:391-3.

14. Stewart RD, Paris PM, Heller MB. Design of a resident in-field experience for an emergency medicine residency curriculum. Ann Emerg Med 1987;16:175-9.

15. Walls RM. Rapid-sequence intubation in head trauma. Ann Emerg Med 1993;22:1008-13.

16. Ravussin P, Freeman J. A new transtracheal catheter for ventilation and resuscitation. Can Anaesth Soc J 1985;32:60-4.

17. Wang HE, Kupas DF, Paris PM, et al. Multivariate Predictors of Failed Prehospital Endotracheal Intubation. Acad Emerg Med 2003;10:717-24.

18. Morton T, Brady S, Clancy M. Difficult airway equipment in English emergency departments. Anaesthesia 2000;55:485-8.

19. Adnet F, Borron SW, Racine SX, et al. The intubation difficulty scale (IDS): proposal and evaluation of a new score characterizing the complexity of endotracheal intubation. Anesthesiology 1997;87:1290-7.
20. Crosby ET, Cooper RM, Douglas MJ, et al. The unanticipated difficult airway with recommendations for management. Can J Anaesth 1998;45:757-76.

21. Davis DP, Hoyt DB, Ochs M, et al. The effect of paramedic rapid sequence intubation on outcome in patients with severe traumatic brain injury. J Trauma 2003;54:444-53.

22. Ochs $M$, Davis D, Hoyt D, et al Paramedic-performed rapid sequence intubation of patients with severe head injuries. Ann Emerg Med 2002;40:159-67.

\section{Address for Correspondence}

Eric Albrecht MD

Service d'Anesthésiologie

Centre Hospitalier Universitaire Vaudois

$\mathrm{BH}-05$, Avenue du Bugnon

1011 Lausanne

Switzerland

Phone $(+41 / 21) 3142047$

e-mail:eric.albrecht@chuv.ch 